\title{
Homocysteine and D-dimer levels and multilayer computed tomography for diagnosing pulmonary artery thromboembolism
}

\author{
Fridon Todua, Manana Akhvlediani, Elena Vorobiova, Giorgi Tsivtsivadze, Anna Baramidze
}

Research Institute of Clinical Medicine, 0112 Tbilisi, Georgia.

Correspondence to: Prof. Manana Akhvlediani, Research Institute of Clinical Medicine, 13, Tevdore Mgvdeli Street, 0112 Tbilisi, Georgia. E-mail: m_akhvlediani2000@hotmail.com

How to cite this article: Todua F, Akhvlediani M, Vorobiova E, Tsivtsivadze G, Baramidze A. Homocysteine and D-dimer levels and multilayer computed tomography for diagnosing pulmonary artery thromboembolism. Vessel Plus 2017;1:38-42.

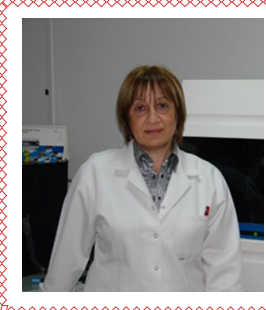

Prof. Manana Akhvlediani is scientific head of Laboratory of Clinical Biochemistry of Research Institute of Clinical Medicine. She received her PhD in biochemistry of atherosclerosis. She is Associated Professor and local head of postgraduate study program in Laboratory Medicine of Georgian Medical University, Tbilisi. She participates in several clinical trials.

\section{Article history:}

Received: 17-10-2016

Accepted: 29-12-2016

Published: 31-03-2017

Key words:

Homocysteine,

D-dimer,

pulmonary embolism

\begin{abstract}
Aim: D-dimer reportedly plays a leading role in diagnosing pulmonary embolism. Additionally, homocysteine is an established risk factor for atherosclerosis, vascular disease, and thrombosis. Herein, the authors aimed to evaluate the diagnostic significance of D-dimer and homocysteine levels, together with multi-detector computed tomography (CT) in suspected pulmonary embolism. Methods: The authors examined patients suffering from conditions and complaints that are typical of pulmonary artery thromboembolism (PATE), such as chest pain, haemoptysis, dyspnoea, tachycardia, arterial hypotension, and signs of vein thrombosis in the inferior limbs. In these patients, PATE was found in different localizations with varying rates of severity. D-dimer levels were measured in patients with suspected PATE using enzyme-linked immunosorbent assays. Homocysteine levels were determined by an enzymatic method. All patients were examined to evaluate the presence of pulmonary embolism by multi-detector CT angiopulmonography. Results: Changes in homocysteine levels can be considered a separate independent factor for PATE diagnostics. The correlation between multi-detector CT angiopulmonography, elevated D-dimer levels, and concomitant hyperhomocysteinemia can be used not only for diagnostics but also for the assessment of the effectiveness of PATE treatment. Conclusion: Multi-detector CT angiopulmonography, D-dimer levels and related hyperhomocysteinemia can serve as significant laboratory markers in the diagnosis and treatment efficacy of PATE.
\end{abstract}

This is an open access article licensed under the terms of Creative Commons Attribution 4.0 International License (https://creativecommons.org/licenses/by/4.0/), which permits unrestricted use, distribution, and reproduction in any medium, as long as the original author is credited and the new creations are licensed under the identical terms.

For reprints contact: service@oaepublish.com

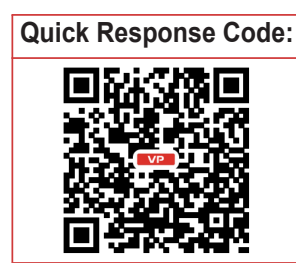




\section{INTRODUCTION}

Pulmonary artery thromboembolism (PATE) is an acute occlusion of the pulmonary trunk, or its branches, with thrombi, formed in the veins of the greater circulation, or in the cavities of the right section of the heart. Modern medical literature uses the term pulmonary embolism. More than 500,000 cases of pulmonary embolism are diagnosed annually. ${ }^{[1-3]}$

Modern diagnostics of PATE have faced substantial difficulties, associated with polymorphism of developing clinical syndromes; suddenness and catastrophic speed of disease development, and the lack of highly informative methods of investigation (perfusive scintigraphy of the lungs, pulmoangiography) in some hospitals..$^{[3-5]}$

The diagnostic problems that need to be solved when patients have suspected PATE are:

(1) to confirm the presence of an embolism;

(2) to determine the exact location of the thromboembolism in pulmonary vessels;

(3) to determine the rate of embolic damage of pulmonary vessel beds;

(4) to assess the haemodynamic state in the greater circulation and the lesser circulation;

(5) to ascertain the source of the embolism and to assess the probability of relapse.

Venous thromboembolism (VTE) is associated with a $5 \%$ to $27 \%$ annual risk of recurrence after the discontinuation of anticoagulant (AC) therapy, and indefinite $A C$ treatment is recommended if the bleeding risk is low-to-moderate. However, in one-third of patients with unprovoked VTE, the risk of recurrence is so low $(<3 \%$ per year) that $A C$ therapy $>3$ months may not be necessary. ${ }^{[4,5]}$

Pulmoangiography is the method of choice in pulmonary embolism diagnostics. However, this examination is associated with discomfort, elevated cost, and the risk of serious complications, typical of invasive procedures. Ventilation-perfusion scintigraphy is widely used at the initial stage of revealing pulmonary embolism, but the validity of the method is limited due to numerous uncertain conclusions. Precise presentation of pulmonary architectonics has become possible since the invention of spiral, and then multilayer scanners, which can provide a higher accuracy of diagnostics. ${ }^{[3,5]}$

It should also be noted that numerous retrospective and prospective investigations have shown a close interdependence between homocysteine (a sulphurcontaining amino acid that is an intermediate product of methionine and cysteine exchange) and cardiovascular diseases, such as venous and arterial thromboses, pulmonary embolism, stroke and myocardial infarction. Hyperhomocysteinemia can suppress a whole range of anti-coagulating mechanisms, which involve the mediation of the vascular endothelium in pathological processes, and as a result, even a moderate increase in homocysteine levels in the blood can cause not only arterial thrombosis and atherosclerosis, but also vein thrombosis. ${ }^{[6-8]}$

Evaluation of D-dimer levels has an active application in current clinical practice, and provides additional information for thrombosis diagnostics (D-dimers are molecules that circulate in the blood during the development of thrombi, which can be resolved under the action of a fibrinolytic system). ${ }^{[9,10]}$

\section{METHODS}

The aim of our investigation was to assess the importance of measuring D-dimer and homocysteine levels, along with the use of multilayer computer tomography $(\mathrm{CT})$, in the diagnosis of patients with suspected PATE.

We examined 54 patients ( 31 males and 23 females) from 18 to 76 years of age, who were suffering from conditions and complaints that are typical of PATE, such as chest pain, haemoptysis, dyspnoea, tachycardia, arterial hypotension and signs of vein thrombosis in the inferior limbs. In 51 patients (94.4\%), PATE was evident in different localizations at varying rates of severity.

A total of 27 healthy persons, with an average age of $52.3 \pm 1.3$ years old, formed a control group. The investigation was randomized, as we did not include patients with normal values of D-dimer, and there were no patients with the above mentioned complains (chest pain, haemoptysis, dyspnoea, tachycardia, arterial hypotension and signs of vein thrombosis in the inferior limbs). All investigations were conducted at the Research Institute of Clinical Medicine.

D-dimer levels were measured in patients with suspected PATE using enzyme-linked immunosorbent assays. Patients with D-dimer levels $>500 \mathrm{ng} / \mathrm{L}$ were subjected to ultrasonic scanning of proximal veins of inferior limbs with compression, and to multilayer CT. Homocysteine levels were measured by an enzymatic method using the biochemical analyser COBAS INTEGRA 400 PLUS (Roche Diagnostics).

Multilayer CT (MLCT) was carried out using the Siemens SOMATOM Sensation 16 Cardiac. During MLCT-pulmoangiography after native examination of 
Table 1: Frequency (\%) of DVT in extremities and PATE in patients at various risk rates

\begin{tabular}{lcccc}
\hline Risk rate & \multicolumn{4}{c}{ DVT/PATE risks (objective test data) } \\
\cline { 2 - 5 } & $\begin{array}{c}\text { Crus vein } \\
\text { thrombosis }\end{array}$ & $\begin{array}{c}\text { Proximal } \\
\text { phlebothrombosis }\end{array}$ & $\begin{array}{c}\text { Clinical } \\
\text { PATE }\end{array}$ & $\begin{array}{c}\text { Mortal } \\
\text { PATE }\end{array}$ \\
\hline High & $40-80$ & $10-30$ & $5-10$ & $1-5$ \\
$\begin{array}{l}\text { Average } \\
\text { (moderate) }\end{array}$ & $10-40$ & $2-10$ & $1-8$ & $0.1-0.7$ \\
Low & $<10$ & $<1$ & $<1$ & $<0.01$ \\
\hline
\end{tabular}

PATE: pulmonary arteria thromboembolism; DVT: deep vein thrombosis

chest organs, we performed contrast enhancement of the pulmonary artery channel, by injecting $70-80 \mathrm{~mL}$ of a contrast substance using an automatic injector at $3 \mathrm{~mL} / \mathrm{s}$; the delay time was 9-11 s, which enabled the initiation of tomography when there was the highest concentration of the contrast substance $(1,220$ Hertz units) in the area of interest, namely the pulmonary artery trunk. During the CT procedure, the patient was instructed to hold his/her breath, or tried to breathe very shallowly. We assessed the condition of pulmonary arteries and their branches up to the sub-segmental level [Table 1].

Ultrasonic scanning was carried out in B-mode, examining common femoral and popliteal veins. The incomplete vascular embarrassment of these veins during compression was considered as the criterion for thrombosis.

All patients were examined with $\mathrm{CT}$ and ultrasonic scanning.

Anticoagulants were prescribed to the patients with thrombosis of proximal deep veins, (ultrasonic scanning and negative CT results). The main indication for analyses was the portion of patients with thrombosis of proximal deep veins and negative CT results. Risk of thromboembolism during the 3 months of attendance was the second indication, if ultrasonic scanning of inferior limbs was not carried out.

Modern methods of variation statistics (Windows 7 , SPSS21 software), performed in Microsoft Excel, were used for statistical processing of obtained results. Sampled simple average $(M)$, simple average of error $(m)$, and average standard deviation $(\sigma)$, were used. Student's criterion $(T)$ was used to define reliability of the difference between simple average values. Comparison of the student's distribution was made. The value of $P$ was $<0.5$ in the groups which we studied. To determine the ratio between variables, we used Pearson's correlation coefficient, where $x_{i}$ and $y_{i}$ are values of compared variables, $\bar{x}$ and $\bar{y}$ are mean values of these variables, and $r$ is the correlation coefficient.
Table 2: Homocysteine and D-dimer levels in patients with pulmonary embolism and control group

\begin{tabular}{lcc|}
\hline $\begin{array}{l}\text { Laboratory } \\
\text { parameters }\end{array}$ & $\begin{array}{c}\text { Patients with pulmonary } \\
\text { embolism }(\boldsymbol{n}=\mathbf{5 4})\end{array}$ & $\begin{array}{c}\text { Control group } \\
(\boldsymbol{n}=\mathbf{2 7})\end{array}$ \\
\hline D-dimer $(\mathrm{ng} / \mathrm{mL})$ & $950.0 \pm 6.0$ & $500.0 \pm 3.0$ \\
$\begin{array}{l}\text { Homocysteine } \\
(\mu \mathrm{mol} / \mathrm{L})\end{array}$ & $26.2 \pm 0.4$ & $9.1 \pm 0.3$ \\
\hline
\end{tabular}

\section{RESULTS}

Our studies showed that hyperhomocysteinemia of up to $26.2 \pm 0.4 \mu \mathrm{mol} / \mathrm{L}$ was found in 50 out of 54 patients $(92.6 \%)$. Homocysteine did not exceed admissible levels and was $8.64 \pm 0.20 \mu \mathrm{mol} / \mathrm{L}$ in 4 patients [Table 2]. It should be noted that homocysteine levels in healthy males and females aged 30 years and above is between 4 and $14 \mu \mathrm{mol} / \mathrm{L}$. A homocysteine concentration of $>15 \mu \mathrm{mol} / \mathrm{L}$ indicates a high risk of developing cardiovascular diseases. ${ }^{[6]}$

Correlation analysis showed a significant positive interdependence between laboratory test results of D-dimer and homocysteine levels with a correlation coefficient of 0.557 .

The series of computer tomograms during MLCT angiography clearly show thrombi in the lumen of pulmonary trunk and its branches of lobar and segmental order.

Thrombi are seen in the lumen as defects of vessel filling, having clear, even outlines of various forms (oval, protruded, irregular form, V-form), dimensions of 2-16 mm and extensions of up to $35 \mathrm{~mm}$. The thrombi have soft-tissue density (35-50 Hertz units), homogeneous structure and partially or completely occlude the damaged vessel.

Multilayer CT pulmoangiography revealed signs of PATE in 19 out of 21 patients (90.5\%), who showed a high probability of disease, evaluated from clinical data. During ultrasound scanning, thrombosis of deep proximal veins was found in 9 out of 19 patients (47.4\%). Only 1 out of 21 patients $(0.5 \%)$ had thrombosis of proximal deep veins and negative CT results. PATE was not revealed by clinical results of CT or ultrasound scanning in 3 patients who had a high probability of PATE, and further angiography also gave negative results. A total of 23 out of 33 patients $(69.6 \%)$ with a low-to-medium probability of PATE had D-dimer levels $>500 \mathrm{ng} / \mathrm{L}$. CT revealed symptoms of PATE in 8 of these 33 patients $(24 \%)$, and thrombosis of proximal deep veins was found in 3 of these 8 patients $(37.5 \%)$ during ultrasound scanning. Only 2 patients $(0.6 \%)$ with low-to-medium PATE probability had thrombosis of maximally deep veins and negative CT results 
[Table 1]. MLCT and pulmoangiography revealed pulmonary infarction of various sizes and localization in $13(24 \%)$ patients, while in $41(76 \%)$ patients, these signs were absent. According to the statistics, PATE causes complications in the form of infarction in 10$30 \%$ of cases.

\section{DISCUSSION}

Pulmonary angiography is a gold standard of PATE diagnostics. However, this method is invasive, causes various complications and is not widely available. In most cases, PATE diagnosis is based on the combination of laboratory tests and radio-diagnostics, such as perfusive radionuclide scintigraphy and echocardiography (cardiac ultrasound), which reveal indirect symptoms of embolism (dilatation of the right ventricle of heart and pulmonary hypertension), observed in severe forms of PATE. CT allows the visualisation of pulmonary arteries and thrombi themselves, as well as the ability to assess structures of mediastinum and parenchyma, which is the main advantage of CT. According to some authors, two thirds of patients with suspected PATE were diagnosed as having aorta dissection, pneumonia, lung carcinoma, and pneumothorax. In addition, the presence of complicated pulmonary embolism (pulmonary infarction, pleural effusion, re-induced vascular picture) is possible. ${ }^{[5,8]}$

With the introduction of high resolution multilayer $\mathrm{CT}$, all the problems associated with the lack of accuracy using spiral CT in PATE diagnostics have been overcome. Using of 14-16 and 64-layer CT decreases the number of unrevealed cases of deseases. Furthermore, visualization of proximal, lobar, and more distal artery branches (at segmental and sub-segmental levels) is provided, revealing peripheral emboli, with precise assessment of their extent being possible. ${ }^{[9]}$

Moreover, to reveal deep vein thromboses, it is possible to combine chest examination with vein CT angiography.

Using reconstructive images, left and right ventricles in axial and four-chamber projections can be assessed and measured. Right ventricle dilation is an unfavourable prognostic factor. ${ }^{[10]}$

We recommend CT examination to be performed at 3 months after diagnosis of PATE, as this period is sufficient for thrombus resolution. Perfusive radionuclide scintigraphy, recommended for a more accurate diagnosis is characterized with high radiation exposure, compared to MLCT, and is not useful for revealing an anatomic picture of the disease. ${ }^{[7,9]}$

On the basis of numerous results, obtained during the investigation of haemostasis and homocysteine, according to which homocysteine can cause a damaging effect on thrombocytes, the interdependence between the rate of D-dimer changes and observed hyperhomocysteinemia becomes evident. Moderate hyperhomocysteinemia, up to $19.0 \mu \mathrm{mol} / \mathrm{L}$ not complicated with thromboembolism was observed in patients with vein thrombosis in inferior limbs. ${ }^{[7,11]}$ If we take considering that even moderate hyperhomocysteinemia can cause oxidative stress (homocysteine oxidation results in the formation of free radicals, which activate the formation of thrombi, in turn resulting in hypercoagulation), then the mechanism of the results observed in patients with thrombosis of proximal deep veins and in thromboembolism of pulmonary artery becomes evident. ${ }^{[11,12]}$

It can be concluded that multilayer CT pulmoangiography is a non-invasive highly informative method, providing a very low level of complications, and is thus indicated for acute PATE diagnostics, and for control of the disease dynamics after anticoagulation treatment. Moderate hyperhomocysteinemia, observed in cases of vein thrombosis of inferior limbs, uncomplicated by PATE, deserves further attention to determine the fate of these patients and the appropriate treatment to provide. The changes in homocysteine levels can be considered as a separate independent factor for PATE diagnostics. The interdependence between D-dimer and concomitant hyperhomocysteinemia can be used not only for diagnostics, but also for the assessment of the effectiveness of PATE treatment.

\section{Authors' contributions}

Concept: F. Todua

Design: M. Akhvlediani

Definition of intellectual content: M. Akhvlediani, E. Vorobiova

Literature search: A. Baramidze

Clinical studies: F. Todua, G. Tsivtsivadze

Data acquisition: G. Tsivtsivadze

Statistical analysis: A. Baramidze

Manuscript preparation, editing and review: $M$. Akhvlediani, E. Vorobiova

\section{Financial support and sponsorship}

None.

\section{Conflicts of interest}

There are no conflicts of interest. 


\section{Patient consent}

\section{All the patients'consent is mentioned in their medical case history.}

\section{Ethics approval}

The study has been approved by the responsible committee on human experimentation of research institute of clinical medicine (protocol 3 25.08.2016).

\section{REFERENCES}

1. Asfar S, Safar H. Homocysteine levels and peripheral arterial occlusive disease: a prospective cohort study and review of the literature. J Cardiovasc Surg (Torino) 2007;48:601-5.

2. Bělohlávek J, Dytrych V, Linhart A. Pulmonary embolism, part I: Epidemiology, risk factors and risk stratification, pathophysiology, clinical presentation, diagnosis and non-thrombotic pulmonary embolism. Exp Clin Cardiol 2013;18:129-38.

3. Kearon C, Akl EA, Comerota AJ, Prandoni P, Bounameaux H, Goldhaber SZ, Nelson ME, Wells PS, Gould MK, Dentali F, Crowther M, Kahn SR; American College of Chest Physicians. Antithrombotic therapy for VTE disease: antithrombotic therapy and prevention of thrombosis, 9th ed: American College of Chest Physicians EvidenceBased Clinical Practice Guidelines. Chest 2012;141:e419S-94S. Erratum in Chest 2012;142:1698-704.

4. Ling IT, Naqvi HA, Siew TK, Loh NK, Ryan GF. SPECT ventilation perfusion scanning with the addition of low-dose CT for the investigation of suspected pulmonary embolism. Intern Med J 2012;42:1257-61.
5. Patel S, Kazerooni EA, Cascade PN. Pulmonary embolism: optimization of small pulmonary artery visualization at multi-detector row CT. Radiology 2003;227:455-60.

6. Refsum H, Smith AD, Ueland PM, Nexo E, Clarke R, McPartlin J, Johnston C, Engbaek F, Schneede J, McPartlin C, Scott JM. Facts and recommendations about total homocysteine determinations: an expert opinion. Clin Chem 2004;50:3-32.

7. Rodger MA, Kahn SR, Wells PS, Anderson DA, Chagnon I, Le Gal G, Solymoss S, Crowther M, Perrier A, White R, Vickars L, Ramsay T, Betancourt MT, Kovacs MJ. Identifying unprovoked thromboembolism patients at low risk for recurrence who can discontinue anticoagulant therapy. CMAJ 2008;179:417-26.

8. Remy-Jardin M, Pistolesi M, Goodman LR, Gefter WB, Gottschalk A, Mayo JR, Sostman HD. Management of suspected acute pulmonary embolism in the era of CT angiography: a statement from the Fleischner Society. Radiology 2007;245:315-29.

9. Robinson K, Mayer E, Jacobsen DW. Homocysteine and coronary artery disease. Cleve Clin J Med 1994;61:438-50.

10. Troy B. Targeted D-dimer testing best for DVT diagnosis. Ann Intern Med 2013;158:93-100.

11. van Belle A, Büller HR, Huisman MV, Huisman PM, Kaasjager K, Kamphuisen PW, Kramer MH, Kruip MJ, Kwakkel-van Erp JM, Leebeek FW, Nijkeuter M, Prins MH, Sohne M, Tick LW; Christopher Study Investigators. Effectiveness of managing suspected pulmonary embolism using an algorithm combining clinical probability, D-dimer testing, and computed tomography. JAMA 2006;295:172-9.

12. Vedovati MC, Becattini C, Agnelli G, Kamphuisen PW, Masotti L, Pruszczyk P, Casazza F, Salvi A, Grifoni S, Carugati A, Konstantinides S, Schreuder M, Golebiowski M, Duranti M. Multidetector CT scan for acute pulmonary embolism: embolic burden and clinical outcome. Chest 2012;142:1417-24. 